\title{
A Study of Flow through a Channel Bounded by a Brinkman Transition Porous Layer
}

\author{
M. S. Abu Zaytoon'1, T. L. Alderson'1, M. H. Hamdan² \\ ${ }^{1}$ Engineering Division, Higher Colleges of Technology, Abu Dhabi Women's Campus, Abu Dhabi, United Arab Emirates \\ ${ }^{2}$ Department of Mathematical Sciences, University of New Brunswick, Saint John, Canada \\ Email:mzaytoon@hct.ac.ae, tim@unb.ca, hamda n@unb.ca
}

How to cite this paper: Abu Zaytoon, M.S. Alderson, T.L. and Hamdan, M.H. (2018) A Study of Flow through a Channel Bounded by a Brinkman Transition Porous Layer. Journal of Applied Mathematics and Physics, 6, 264-282.

https://doi.org/10.4236/jamp.2018.61025

Received: November 24, 2017

Accepted: January 26, 2018

Published: January 29, 2018

Copyright (c) 2018 by authors and Scientific Research Publishing Inc. This work is licensed under the Creative Commons Attribution International License (CC BY 4.0).

http://creativecommons.org/licenses/by/4.0/

\begin{abstract}
Flow through a channel bounded by a porous layer is considered when a transition layer exists between the channel and the medium. The variable permeability in the transition layer is chosen such that Brinkman's equation governing the flow reduces to a generalized inhomogeneous Airy's differential equation. Solution to the resulting generalized Airy's equation is obtained in this work and solution to the flow through the transition layer, of the same configuration, reported in the literature, is recovered from the current solution.
\end{abstract}

\section{Keywords}

Transition Layer, Generalized Airy's Equation

\section{Introduction}

In their recent study, Nield and Kuznetsov [1] provided elegant analysis of the mechanics of a transition layer between a Darcy porous layer and a Navier-Stokes channel. In the transition layer, they employed Brinkman's equation with variable permeability and provided exact solution of the flow in terms of Airy's functions. Rooted in the work of Nield [2], the concept of the transition layer has been implemented and validated in the analysis provided by number of other authors over the last two decades (cf. Goyeau et al. [3]; Goharzadeh et al. [4]; Parvazinia et al. [5]; Chandesris and Jamet [6]; Hill and Straughan [7]; Duman and Shavit [8]), thus illustrating further usefulness of Brinkman's equation in the study of flow over porous layers. Furthermore, the Nield and Kuznetsov approach [1] continues to receive attention, and has been implemented by a number of authors in their solution of problems involving the transition zone (cf. Tao et al. [9] and the references therein). In addition to providing an impetus to 
the transition zone approach, the work of Nield and Kuznetsov [1] initiated a number of firsts that include:

1) Introducing and reviving the implementation of classical integral functions in the porous media literature (as witnessed by their use of Airy's differential equation and the Airy functions in providing an analytical solution to the flow in the transition zone);

2) Introducing a new integral function, $\mathrm{Ni}(\mathrm{x})$, to facilitate solution to the inhomogeneous Airy's differential equation. This function is better known as the Nield-Kuznetsov function, and has been studied extensively by Hamdan and Kamel [10], as it proved to possess favorable mathematical properties that include its serving as a solution to a number of well-known differential equations, such as Langer's comparison differential equation, Hamdan and Kamel [10].

3) Initiating non-traditional models of permeability variations in porous media. While the classical use of elementary mathematical functions has served the subject matter well, the use of special functions in advancing the topic represents a new generation of models the computations of which is no longer a formidable task.

Many excellent reviews of flow through and over porous layers are available in the literature (cf. Nield and Bejan [11]; Nield [12]), and it is not our intention to revisit appropriate models and conditions at the interface between a fluid and a porous layer. Rather, our intention in this work is to revisit the problem considered by Nield and Kuznetsov [1] and provide an alternative variable permeability model that reduces Brinkman's equation in the transition zone to a generalized Airy's differential equation (in the sense introduced by Swanson and Headley [13]). We will present an analytical solution in terms of the generalized Airy's functions, and provide computations using Maple's built-in functions. The solution provided by Nield and Kuznetsov [1] is then compared with a special case of the current formulation, which shows agreement between solutions obtained. It is believed that the current approach will provide extended flexibility in modelling permeability variations in the transition zone.

\section{Problem Formulation}

We consider fluid flow through a channel consisting of three layers, as shown in Figure 1. The first layer consists of free-space, bounded below be a solid, impermeable wall and bounded above by the transition porous layer, and is occupied by a fluid whose flow is governed by Navier-Stokes equations. The second (middle) layer is a porous medium with variable permeability taken as the reciprocal of a polynomial of degree $n$. This is the transition layer that bounds the free-space channel from above, and is saturated with fluid whose flow is governed by Brinkman's equation. The third layer is a porous medium with constant permeability that is bounded from above by a solid, impermeable wall, and bounded from below by the variable permeability transition layer. Flow in the third layer is governed by Brinkman's equation, and the flow through the three 


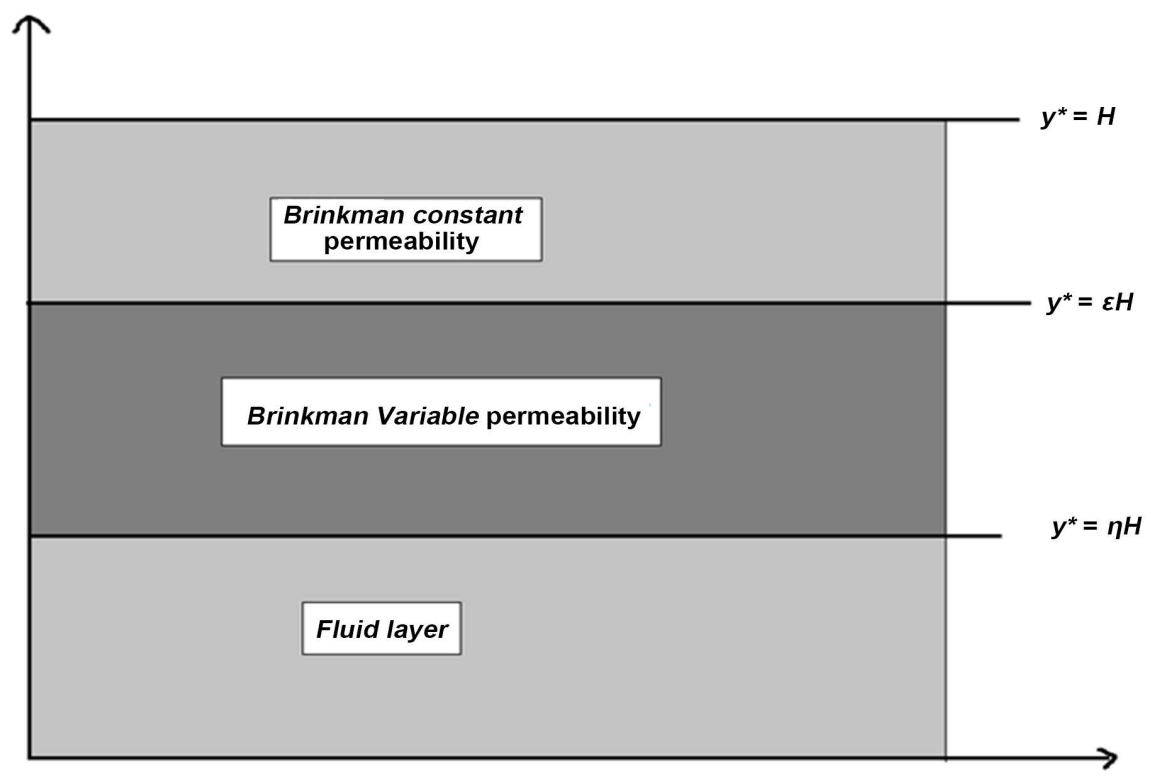

Figure 1. Representative sketch of a channel bounded by a transition porous layer.

layers is driven by a common pressure gradient. Permeability distribution in the configuration of Figure 1 is given as follows, where we take $K_{0}$ as a constant reference permeability.

In the lower region, or Region 1 (the fluid zone):

$$
K_{1} \rightarrow \infty \text { for } 0<y^{*}<\eta H .
$$

In the middle region, or Region 2:

$$
K_{2}=\frac{K_{0}[(\xi-\eta) H]^{n}}{\left(y^{*}-\eta H\right)^{n}} ; \text { for } \eta H<y^{*}<\xi H .
$$

In the upper region, or Region 3:

$$
K_{3}=K_{0} ; \text { for } \xi H<y^{*}<H .
$$

The boundary value problem at hand can be formulated as follows:

$$
\begin{gathered}
\mu_{1} \frac{\mathrm{d}^{2} u_{1}^{*}}{\mathrm{~d} y^{* 2}}+G=0 \quad \text { for } 0<y^{*}<\eta H . \\
\mu_{e 2} \frac{\mathrm{d}^{2} u_{2}^{*}}{\mathrm{~d} y^{* 2}}-\frac{\mu}{K_{2}} u_{2}+G=0 \quad \text { for } \eta H<y^{*}<\xi H . \\
\mu_{e_{3}} \frac{\mathrm{d}^{2} u_{3}^{*}}{\mathrm{~d} y^{* 2}}-\frac{\mu}{K_{3}} u_{3}^{*}+G=0 \quad \text { for } \xi H<y^{*}<H .
\end{gathered}
$$

In the above equations, $G=-\frac{\mathrm{d} p}{\mathrm{~d} x}$ is the constant pressure gradient. For $i=1,2,3$, with $i=1$ referring to layer $1, i=2$ referring to layer 2 , and $i=3$ referring to layer 3, the quantities in Equations (4), (5), and (6): $u_{i}^{*}, K_{i}, \mu_{i}$, and $\mu_{e i}$ denote the velocity, permeability, base fluid viscosity, and effective viscosity, respectively, in layer $i$. 
Following Nield and Kozentsov, [1], we introduce the following dimensionless variables, where $D a$ refers to Darcy number: $y=\frac{y^{*}}{H} ; D a=\frac{K_{0}}{H^{2}} ; u_{i}=\frac{\mu_{i}}{G H^{2}} u_{i}^{*}$ for $i=1,2,3$; and $M_{i}=\frac{\mu_{e i}}{\mu_{i}}$ for $i=2,3$.

The permeability distributions, $K_{1}, K_{2}$ and $K_{3}$ can be written in the following dimensionless form in terms of Darcy number, Da:

$$
\begin{gathered}
K_{1}^{*}=\frac{K_{1}}{H^{2}} \rightarrow \infty \\
K_{2}^{*}=\frac{K_{2}}{H^{2}}=D a\left(\frac{\xi-\eta}{y-\eta}\right)^{n} \\
K_{3}^{*}=\frac{K_{3}}{H^{2}}=D a .
\end{gathered}
$$

Equations (4), (5), and (6) take the following forms, respectively:

$$
\begin{gathered}
\frac{\mathrm{d}^{2} u_{1}}{\mathrm{~d} y^{2}}+1=0 ; \quad 0<y<\eta . \\
M_{2} \frac{\mathrm{d}^{2} u_{2}}{\mathrm{~d} y^{2}}-\frac{H^{2}}{K_{2}} u_{2}+1=0 ; \quad \eta<y<\xi . \\
M_{3} \frac{\mathrm{d}^{2} u_{3}}{\mathrm{~d} y^{2}}-\frac{H^{2}}{K_{3}} u_{3}+1=0 ; \quad \xi<y<1
\end{gathered}
$$

and, upon substituting the permeability distributions, (7)-(9), in equations (10)-(12), we get

$$
\begin{gathered}
\frac{\mathrm{d}^{2} u_{1}}{\mathrm{~d} y^{2}}+1=0 ; \quad 0<y<\eta . \\
\frac{\mathrm{d}^{2} u_{2}}{\mathrm{~d} y^{2}}-\frac{1}{M_{2} D a(\xi-\eta)^{n}}(y-\eta)^{n} u_{2}+\frac{1}{M_{2}}=0 ; \quad \eta<y<\xi . \\
\frac{\mathrm{d}^{2} u_{3}}{\mathrm{~d} y^{2}}-\frac{1}{D a M_{3}} u_{3}+\frac{1}{M_{3}}=0 ; \quad \text { for } \xi<y<1 .
\end{gathered}
$$

Equation (13), (14) and (15) must be solved subject to the following boundary and matching conditions

$$
\begin{gathered}
u_{1}=0 \text { at } y=0 \\
u_{1}=u_{2} \text { at } y=\eta \\
\frac{\mathrm{d} u_{1}}{\mathrm{~d} y}=\vartheta_{1} \frac{\mathrm{d} u_{2}}{\mathrm{~d} y} \text { at } y=\eta . \\
u_{2}=u_{3} \text { at } y=\xi . \\
\vartheta_{2} \frac{\mathrm{d} u_{2}}{\mathrm{~d} y}=\frac{\mathrm{d} u_{3}}{\mathrm{~d} y} \text { at } y=\xi . \\
u_{3}=0 \text { at } y=1,
\end{gathered}
$$


where $\vartheta_{1}=\frac{\mu_{2}}{\mu_{1}}$ and $\vartheta_{2}=\frac{\mu_{2}}{\mu_{3}}$ are the viscosity ratios.

Now, letting

$$
\begin{gathered}
\tau_{n}=\frac{1}{\sqrt[n+2]{M_{2} D a(\xi-\eta)^{n}}} \\
\lambda_{3}=\frac{1}{\sqrt{M_{3} D a}}
\end{gathered}
$$

Equation (14) and Equation (15) become, respectively:

$$
\begin{gathered}
\frac{\mathrm{d}^{2} u_{2}}{\mathrm{~d} y^{2}}-\left(\tau_{n}\right)^{n+2}(y-\eta)^{n}+\frac{1}{M_{2}}=0 \\
\frac{\mathrm{d}^{2} u_{3}}{\mathrm{~d} y^{2}}-\lambda_{3}^{2} u_{3}+\frac{1}{M_{3}}=0 .
\end{gathered}
$$

General solutions for Equation (13) and Equation (20) are given, respectively, by

$$
\begin{gathered}
u_{1}(y)=c_{1} y+d_{1}-\frac{y^{2}}{2} \\
u_{3}(y)=c_{3} \exp \left(\lambda_{3} y\right)+d_{3} \exp \left(-\lambda_{3} y\right)+\frac{1}{M_{3} \lambda_{3}^{2}} .
\end{gathered}
$$

In order to solve Equation (19), we first use the following transformation $\tilde{y}=\tau_{n}(y-\eta)$ and write $u_{2}(y) \equiv U_{2}(\tilde{y})$. Equation (19) then becomes:

$$
\frac{\mathrm{d}^{2} U_{2}(\tilde{y})}{\mathrm{d} \tilde{y}^{2}}-(\tilde{y})^{n} U_{2}(\tilde{y})+\frac{1}{M_{2}\left(\tau_{n}\right)^{2}}=0
$$

Equation (23) is the generalized inhomogeneous Airy's differential equation. A fundamental pair of linearly independent solutions for the homogeneous part are the generalized Airy's functions $A_{n}(\tilde{y})$ and $B_{n}(\tilde{y})$, (cf. Swanson and Headley [13]), defined respectively by

$$
\begin{aligned}
& A_{n}(\tilde{y})=\frac{2 p}{\pi} \sin (p \pi)(\tilde{y})^{1 / 2} K_{p}(\zeta) \\
& B_{n}(\tilde{y})=(p \tilde{y})^{\frac{1}{2}}\left(I_{-p}(\zeta)+I_{p}(\zeta)\right)
\end{aligned}
$$

The terms $I_{p}$ and $K_{p}$ are the modified Bessel functions defined as:

$$
\begin{gathered}
I_{p}(\zeta)=i^{-p} J_{p}(i \zeta)=\sum_{m=1}^{\infty} \frac{1}{m ! \Gamma(m+p+1)}\left(\frac{\zeta}{2}\right)^{2 m+p} \\
K_{p}(\zeta)=\frac{\pi}{2} \frac{\left(I_{-p}(\zeta)-I_{p}(\zeta)\right)}{\sin (p \pi)}
\end{gathered}
$$

with $p=\frac{1}{n+2}, \zeta=2 p Y^{\frac{1}{2 p}}$, and $\Gamma$ is the gamma function.

Solution to the homogeneous part of (23) is thus given by

$$
U_{2 h}(\tilde{y})=c_{2} A_{n}(\tilde{y})+d_{2} B_{n}(\tilde{y}) .
$$


We find it convenient to introduce the following integral function:

$$
Z_{n}(\tilde{y})=A_{n}(\tilde{y}) \int_{0}^{\tilde{y}} B_{n}(t) \mathrm{d} t-B_{n}(\tilde{y}) \int_{0}^{\tilde{y}} A_{n}(t) \mathrm{d} t .
$$

The function $Z_{n}(\tilde{y})$ reduces to the Nield-Kuznetsov function $N_{i}(\tilde{y})$ when $n=1$.

The Wronskian of $A_{n}(Y)$ and $B_{n}(Y)$ is given by:

$$
W\left(A_{n}(\tilde{y}), B_{n}(\tilde{y})\right)=\frac{2}{\pi} p^{\frac{1}{2}} \sin (p \pi)
$$

and general solution of (23) is expressed, using variation of parameters, as:

$$
U_{2}(\tilde{y})=c_{2} A_{n}(\tilde{y})+d_{2} B_{n}(\tilde{y})+\sigma_{n} Z_{n}(\tilde{y})
$$

where $p=\frac{1}{n+2}$ and $\sigma_{n}=\frac{\pi}{2 \sqrt{p} \sin (p \pi) M_{2}\left(\tau_{n}\right)^{2}}$.

Upon substituting $\tilde{y}=\tau_{n}(y-\eta), u_{2}(y) \equiv U_{2}(\tilde{y})$, and

$\sigma_{n}=\frac{\pi}{2 \sqrt{p} \sin (p \pi) M_{2}\left(\tau_{n}\right)^{2}}$, in (28) we obtain the following general solution to Equation (14):

$$
\begin{aligned}
u_{2}(y)= & c_{2} A_{n}\left(\tau_{n}(y-\eta)\right)+d_{2} B_{n}\left(\tau_{n}(y-\eta)\right) \\
& +\frac{\pi}{2 \sqrt{p} \sin (p \pi) M_{2}\left(\tau_{n}\right)^{2}} Z_{n}\left(\tau_{n}(y-\eta)\right)
\end{aligned}
$$

Derivatives of the functions $A_{n}(\tilde{y}), B_{n}(\tilde{y})$ and $Z_{n}(\tilde{y})$ are given by:

$$
\begin{gathered}
A_{n}^{\prime}(\tilde{y})=-\frac{2 p}{\pi}[\sin p y] \tilde{y}^{\frac{n+1}{2}} K_{p-1}(\zeta) \\
B_{n}^{\prime}(\tilde{y})=p^{\frac{1}{2}} \tilde{y}^{\frac{n+1}{2}}\left[I_{1-p}(\zeta)+I_{p-1}(\zeta)\right] \\
Z_{n}^{\prime}(\tilde{y})=A_{n}^{\prime}(\tilde{y}) \int_{0}^{\tilde{y}} B_{n}(t) \mathrm{d} t-B_{n}^{\prime}(\tilde{y}) \int_{0}^{\tilde{y}} A_{n}(t) \mathrm{d} t .
\end{gathered}
$$

Shear stress expressions across the layers are obtained from Equations (21), (22) and (32), and take the following form:

$$
\begin{gathered}
\frac{\mathrm{d} u_{1}}{\mathrm{~d} y}=c_{1}-y \\
\frac{\mathrm{d} u_{2}}{\mathrm{~d} y}=c_{2} \tau_{n} A_{n}^{\prime}\left(\tau_{n}(y-\eta)\right)+d_{2} \tau_{n} B_{n}^{\prime}\left(\tau_{n}(y-\eta)\right) \\
+\frac{\pi}{2 \sqrt{p} \sin (p \pi) M_{2} \tau_{n}} Z_{n}^{\prime}\left(\tau_{n}(y-\eta)\right) \\
\frac{\mathrm{d} u_{3}}{\mathrm{~d} y}=c_{3} \lambda_{3} \exp \left(\lambda_{3} y\right)-d_{3} \lambda_{3} \exp \left(-\lambda_{3} y\right) .
\end{gathered}
$$

Upon using boundary and interfacial conditions, (16a)-(16f), in Equations (21), (22) and (32), we obtain the following system of linear equations that is to be solved for the arbitrary constants $c_{1}, d_{1}, c_{2}, d_{2}, c_{3}, d_{3}$ :

$$
d_{1}=0
$$




$$
\begin{gathered}
c_{1} \eta+d_{1}-c_{2} A_{n}(0)-d_{2} B_{n}(0)=\frac{\eta^{2}}{2} \\
c_{1}-c_{2} \vartheta_{1} \tau_{n} A_{n}^{\prime}(0)-d_{2} \vartheta_{1} \tau_{n} B_{n}^{\prime}(0)=\eta \\
c_{2} A_{n}\left(\tau_{n}(\xi-\eta)\right)+d_{2} B_{n}\left(\tau_{n}(\xi-\eta)\right)-c_{3} \exp \left(\lambda_{3} \xi\right)-d_{3} \exp \left(-\lambda_{3} \xi\right) \\
=\frac{1}{M_{3} \lambda_{3}^{2}}-\frac{\pi}{2 \sqrt{p} \sin (p \pi) M_{2}\left(\tau_{n}\right)^{2}} Z_{n}\left(\tau_{n}(\xi-\eta)\right) \\
c_{2} \vartheta_{2} \tau_{n} A_{n}^{\prime}\left(\tau_{n}(\xi-\eta)\right)+d_{2} \vartheta_{2} \tau_{n} B_{n}^{\prime}\left(\tau_{n}(\xi-\eta)\right)-\lambda_{3} c_{3} \exp \left(\lambda_{3} \xi\right)+\lambda_{3} d_{3} \exp \left(-\lambda_{3} \xi\right) \\
=-\frac{\vartheta_{2} \pi}{2 \sqrt{p} \sin (p \pi) M_{2} \tau_{n}} Z_{n}^{\prime}\left(\tau_{n}(\xi-\eta)\right) \\
c_{3} \exp \left(\lambda_{3}\right)+d_{3} \exp \left(-\lambda_{3}\right)=\frac{-1}{M_{3} \lambda_{3}^{2}}
\end{gathered}
$$

Linear Equations (39)-(44) are cast in the following matrix-vector form

$$
M x=c,
$$

where:

$$
\boldsymbol{M}=\left(\begin{array}{cccccc}
0 & 1 & 0 & 0 & 0 & 0 \\
\eta & 1 & -A_{n}(0) & -B_{n}(0) & 0 & 0 \\
1 & 0 & -\vartheta_{1} \tau_{n} A_{n}^{\prime}(0) & -\vartheta_{1} \tau_{n} B_{n}(0) & 0 & 0 \\
0 & 0 & A_{n}^{\prime}\left(\tau_{n}(\xi-\eta)\right) & B_{n}\left(\tau_{n}(\xi-\eta)\right) & -\exp \left(\lambda_{3} \xi\right) & -\exp \left(-\lambda_{3} \xi\right) \\
0 & 0 & \vartheta_{2} \tau_{n} A_{n}^{\prime}\left(\tau_{n}(\xi-\eta)\right) & \vartheta_{2} \tau_{n} B_{n}^{\prime}\left(\tau_{n}(\xi-\eta)\right) & -\lambda_{3} \exp \left(\lambda_{3} \xi\right) & \lambda_{3} \exp \left(-\lambda_{3} \xi\right) \\
0 & 0 & 0 & 0 & \exp \left(\lambda_{3}\right) & \exp \left(-\lambda_{3}\right)
\end{array}\right)
$$

$$
\begin{aligned}
& \boldsymbol{x}=\left[\begin{array}{l}
c_{1} \\
d_{1} \\
c_{2} \\
d_{2} \\
c_{3} \\
d_{3}
\end{array}\right] \\
& \boldsymbol{c}=\left(\begin{array}{c}
0 \\
\frac{\eta^{2}}{2} \\
\frac{1}{M_{3} \lambda_{3}^{2}}-\frac{\pi}{2 \sqrt{p} \sin (p \pi) M_{2}\left(\tau_{n}\right)^{2}} Z_{n}\left(\tau_{n}(\xi-\eta)\right) \\
-\frac{\vartheta_{2} \pi}{2 \sqrt{p} \sin (p \pi) M_{2} \tau_{n}} Z_{n}^{\prime}\left(\tau_{n}(\xi-\eta)\right) \\
\frac{-1}{M_{3} \lambda_{3}^{2}}
\end{array}\right) .
\end{aligned}
$$

In solving the above linear system, we make use of the following values of the generalized functions $A_{n}, B_{n}$ and $Z_{n}$, and their first derivatives at zero ( $c f$. [13]), wherein $\Gamma$ is the Gamma function: 


$$
\begin{gathered}
A_{n}(0)=\frac{p^{1-p}}{\Gamma(1-p)} \\
B_{n}(0)=\frac{p^{\frac{1}{2}-p}}{\Gamma(1-p)} \\
A_{n}^{\prime}(0)=-\frac{p^{p}}{\Gamma(p)} \\
B_{n}^{\prime}(0)=\frac{p^{p-\frac{1}{2}}}{\Gamma(p)} \\
Z_{n}(0)=0 \\
Z_{n}^{\prime}(0)=0 .
\end{gathered}
$$

\section{Results and Analysis}

Results have been obtained for a range of values of $n$ and the range of $D a=1 ; 0.1$; $0.001 ; 0.0001$; and 0.00001 in order to illustrate the effects of changing $n$ and $D a$ on the generalized functions, on the permeability function, on the arbitrary constants, and on the velocity profiles. Thick and thin transition layers are considered. In particular, we choose $\eta=1 / 3, \xi=2 / 3$ and $\eta=1 / 4, \xi=3 / 4$ for thick transition layer, and $\eta=0.49, \xi=0.51$ for thin layer.

Values of $p=\frac{1}{n+2}, A_{n}, A_{n}^{\prime}, B_{n}, B_{n}^{\prime}, Z_{n}, Z_{n}^{\prime}, \sigma_{n}$ and $\tau_{n}$ at 0 and at other specified points for different values of $n$ and $D a$, have been computed using Maple. These values are important in the calculation of the arbitrary constants in the matrix-vector Equations (45), and in the determination and plotting of permeability functions and velocity profiles. Computational results are most accurate when $\mathrm{Da}=1 ; 0.1$; and 0.001 .

\section{Dimensionless Permeability Distributions}

For the sake of graphs we find it more convenient to plot the reciprocal of the dimensionless permeability functions (7)-(9), namely

$$
\begin{gathered}
\frac{1}{K_{1}^{*}}=0 \quad \text { for } 0<y<\eta . \\
\frac{1}{K_{2}^{*}(y)}=\frac{1}{D a}\left(\frac{y-\eta}{\xi-\eta}\right)^{n} ; \quad \text { for } \eta<y<\xi . \\
\frac{1}{K_{1}^{*}}=\frac{1}{D a} \quad \text { for } \xi<y<1 .
\end{gathered}
$$

Dependence of the permeability distributions on the value of $n$ is illustrated in Figure 2, which shows the reciprocal of the dimensionless permeability across the three layers for $n=1,2,3$ and 5 , for $\eta=\frac{1}{3}, \xi=\frac{2}{3}$, and $D a=1$. Figure 2 


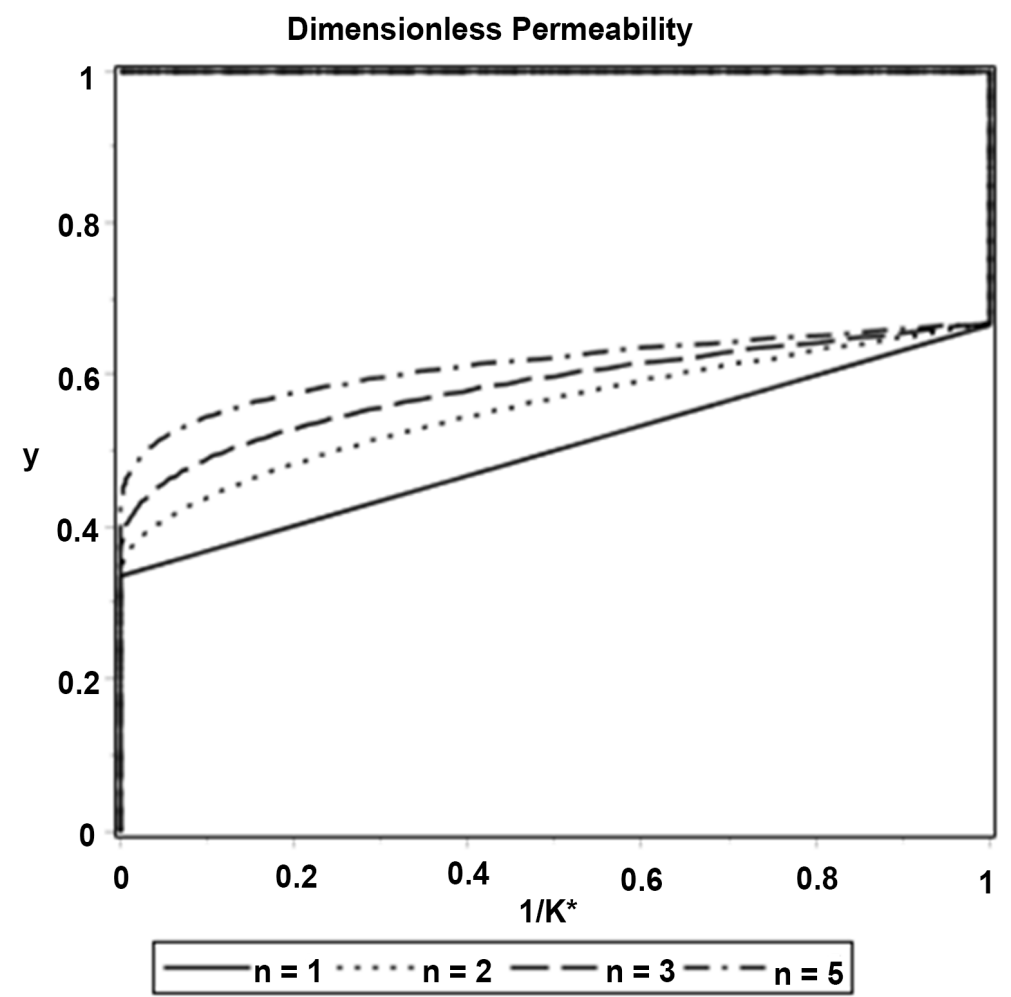

Figure 2. Dimensionless permeability distributions for $\eta=\frac{1}{3}, \xi=\frac{2}{3}, D a=1$, and different $n$.

demonstrates the increase of the reciprocal permeability of the transition layer (or decrease of permeability) with increasing $n$.

\section{Velocity at the Interfaces}

At the lower and upper interfaces, $y=\eta$ and $y=\xi$, respectively, between layers, velocity expressions are obtained from Equations (21), (22), and (32), and take the form

$$
\begin{gathered}
u_{1}(\eta)=c_{1} \eta+d_{1}-\frac{\eta^{2}}{2} \\
u_{2}(\eta)=c_{2} A_{n}(0)+d_{2} B_{n}(0) \\
u_{2}(\xi)=c_{2} A_{n}\left(\tau_{n}(\xi-\eta)\right)+d_{2} B_{n}\left(\tau_{n}(\xi-\eta)\right) \\
+\frac{\pi}{2 \sqrt{p} \sin (p \pi) M_{2}\left(\tau_{n}\right)^{2}} Z_{n}\left(\tau_{n}(\xi-\eta)\right) \\
u_{3}(\xi)=c_{3} \exp \left(\lambda_{3} \xi\right)+d_{3} \exp \left(-\lambda_{3} \xi\right)+\frac{1}{M_{3} \lambda_{3}^{2}} .
\end{gathered}
$$

Velocity at the lower and upper interfaces for different middle layer thickness, different values of $D a$, and for $n=1$ and $n=2$ are given in Table 1 and Table 2, which furnish the following observations: 
Table 1. Velocity for $n=1$ at the upper and lower interface.

\begin{tabular}{llllll}
\hline \multicolumn{1}{c}{} & $\mathrm{Da}$ & $\mathrm{Da}=1$ & $\mathrm{Da}=0.1$ & $\mathrm{Da}=0.01$ & $\mathrm{Da}=0.001$ \\
\hline$\eta=1 / 3$ & $u_{1}(\eta)=u_{2}(\eta)$ & 0.1068281 & 0.08214691 & 0.03908309 & 0.01714995 \\
$\xi=2 / 3$ & $u_{2}(\xi)=u_{3}(\xi)$ & 0.1046975 & 0.06834714 & 0.01327264 & 0.00107072 \\
$\eta=4$ & $u_{1}(\eta)=u_{2}(\eta)$ & 0.09037786 & 0.07064158 & 0.03455354 & 0.01513239 \\
$\xi=3 / 4$ & $u_{2}(\xi)=u_{3}(\xi)$ & 0.0883398 & 0.0574775 & 0.0110710 & 0.00101 \\
$\eta=0.49$ & $u_{1}(\eta)=u_{2}(\eta)$ & 0.1189297 & 0.08497432 & 0.0308578 & 0.01043350 \\
$\xi=0.51$ & $u_{2}(\xi)=u_{3}(\xi)$ & 0.1186918 & 0.0833988 & 0.02721074 & 0.00630965 \\
\hline
\end{tabular}

Table 2. Velocity for $n=2$ at the upper and lower interface.

\begin{tabular}{llcccc}
\hline & & $D a=1$ & $D a=0.1$ & $D a=0.01$ & $D a=0.001$ \\
\hline$\eta=1 / 3$ & $u_{1}(\eta)=u_{2}(\eta)$ & 0.1078735 & 0.08819101 & 0.04952703 & 0.02688473 \\
$\xi=2 / 3$ & $u_{2}(\xi)=u_{3}(\xi)$ & 0.1057217 & 0.07335809 & 0.01652092 & 0.0012043 \\
$\eta=4$ & $u_{1}(\eta)=u_{2}(\eta)$ & 0.0915251 & 0.07740496 & 0.04608594 & 0.0252177 \\
$\xi=3 / 4$ & $u_{2}(\xi)=u_{3}(\xi)$ & 0.0894578 & 0.0628103 & 0.0134685 & 0.00108 \\
$\eta=0.49$ & $u_{1}(\eta)=u_{2}(\eta)$ & 0.119023 & 0.0854829 & 0.03166939 & 0.01131475 \\
$\xi=0.51$ & $u_{2}(\xi)=u_{3}(\xi)$ & 0.1187851 & 0.08390017 & 0.02796018 & 0.00694503 \\
\hline
\end{tabular}

1) Computations render reasonable results for $D a$ as low as 0.001 when $n=1$. Inaccuracies start creeping when $D a<0.001$. For $n=2$, results are reasonable for $D a$ as low as 0.0001 and inaccuracies creep when $D a<0.0001$. This behavior may be attributed to both round-off errors for small $D a$ and inaccuracies in evaluation of Airy's functions for small $D a$. This behavior persists for thick layers, and is less noticeable for thin middle layer calculations.

2) Computations of velocity at the lower interface using expressions (58) and (59) agree up to within seven significant digits. The same is true for computations of velocity at the upper interface using expressions (60) and (61). This is indicative of appropriate matching of the velocity at the interfaces, used in this work.

3) For a given $D a$, velocity at the lower interface decreases with increasing middle layer thickness. Similarly, velocity at the upper interface decreases with increasing middle layer thickness. This behavior persists for both $n=1$ and $n=$ 2.

4) For a given middle layer thickness, velocity at each of the lower and upper interfaces increases with increasing $D a$. This is expected, as $D a$ is defined as a dimensionless reference permeability and accompanied with increasing permeability is a velocity increase.

5) The effect of increasing $n$ on the velocity at the interfaces for a given thickness and $D a$ is that the velocity at each interface increases with increasing $n$. This is true for both thin and thick transition layers, and for the range of Da used. 


\section{Shear Stress at the Interfaces}

At the lower and upper interfaces, $y=\eta$ and $y=\xi$, respectively, between layers, shear stress expressions are obtained from Equations (36), (37), and (38), and take the form

$$
\begin{gathered}
\frac{\mathrm{d} u_{1}(\eta)}{\mathrm{d} y}=c_{1}-\eta \\
\frac{\mathrm{d} u_{2}(\eta)}{\mathrm{d} y}=c_{2} \tau_{n} A_{n}^{\prime}(0)+d_{2} \tau_{n} B_{n}^{\prime}(0) \\
\frac{\mathrm{d} u_{2}(\xi)}{\mathrm{d} y}=c_{2} \tau_{n} A_{n}^{\prime}\left(\tau_{n}(\xi-\eta)\right)+d_{2} \tau_{n} B_{n}^{\prime}\left(\tau_{n}(\xi-\eta)\right) \\
+\frac{\pi}{2 \sqrt{p} \sin (p \pi) M_{2} \tau_{n}} Z_{n}^{\prime}\left(\tau_{n}(\xi-\eta)\right) \\
\frac{\mathrm{d} u_{3}(\xi)}{\mathrm{d} y}=c_{3} \lambda_{3} \exp \left(\lambda_{3} \xi\right)-d_{3} \lambda_{3} \exp \left(-\lambda_{3} \xi\right) .
\end{gathered}
$$

Shear stress at the lower and upper interfaces for different middle layer thickness, different values of $D a$, and for $n=1$ and $n=2$ are given in Table 3 and Table 4, which furnish the following observations:

1) Computations render reasonable results for $D a$ as low as 0.001 when $n=1$ and $n=2$. Inaccuracies start creeping when $D a<0.001$. This behavior may be attributed to the inaccuracies reported earlier when computing velocity at the interfaces using Maple's built-in functions. When dealing with a thin transition layer, results are accurate for as low as $D a=0.00001$.

2) Computations of shear stress at the lower interface using expressions (62) and (63) agree up to within a minimum of five significant digits. The same is true for computations of velocity at the upper interface using expressions (64) and (65).

\begin{tabular}{|c|c|c|c|c|c|}
\hline$D a$ & & $D a=1$ & $D a=0.1$ & $D a=0.01$ & $D a=0.001$ \\
\hline$\eta=1 / 3$ & $\frac{\mathrm{d} u_{1}}{\mathrm{~d} y}(\eta)=\frac{\mathrm{d} u_{2}}{\mathrm{~d} y}(\eta)$ & 0.1538176 & 0.07977407 & -0.04941739 & -0.1152168 \\
\hline$\xi=2 / 3$ & $\frac{\mathrm{d} u_{2}}{\mathrm{~d} y}(\xi)=\frac{\mathrm{d} u_{3}}{\mathrm{~d} y}(\xi)$ & -0.1604999 & -0.1231173 & -0.0399533 & -0.00225 \\
\hline$\eta=1 / 4$ & $\frac{\mathrm{d} u_{1}}{\mathrm{~d} y}(\eta)=\frac{\mathrm{d} u_{2}}{\mathrm{~d} y}(\eta)$ & 0.2365115 & 0.1575664 & 0.01321413 & -0.06447045 \\
\hline$\xi=3 / 4$ & $\frac{\mathrm{d} u_{2}}{\mathrm{~d} y}(\xi)=\frac{\mathrm{d} u_{3}}{\mathrm{~d} y}(\xi)$ & -0.2363372 & -0.1570521 & -0.027384 & -0.003 \\
\hline$\eta=0.49$ & $\frac{\mathrm{d} u_{1}}{\mathrm{~d} y}(\eta)=\frac{\mathrm{d} u_{2}}{\mathrm{~d} y}(\eta)$ & -0.002286399 & -0.071583 & -0.1820249 & -0.2237071 \\
\hline$\xi=0.51$ & $\frac{\mathrm{d} u_{2}}{\mathrm{~d} y}(\xi)=\frac{\mathrm{d} u_{3}}{\mathrm{~d} y}(\xi)$ & -0.02109832 & -0.08318887 & -0.1736158 & -0.167906 \\
\hline
\end{tabular}

3) For a given $D a$, the absolute value of the shear stress at the lower interface increases with increasing transition layer thickness. Similarly, at the upper interface. This behavior persists for both $n=1$ and $n=2$.

Table 3. Shear stress at the upper and lower interfaces for $\mathrm{n}=1$. 
Table 4. Shear stress at the upper and lower interfaces for $n=2$.

\begin{tabular}{llcccc}
\hline$D a$ & & $D a=1$ & $D a=0.1$ & $D a=0.01$ & $D a=0.001$ \\
\hline $\begin{array}{l}\eta=1 / 3 \\
\xi=2 / 3\end{array}$ & $\frac{\mathrm{d} u_{1}}{\mathrm{~d} y}(\eta)=\frac{\mathrm{d} u_{2}}{\mathrm{~d} y}(\eta)$ & 0.1569537 & 0.09790637 & -0.01808557 & -0.08601249 \\
& $\frac{\mathrm{d} y}{\mathrm{~d} u_{1}}(\xi)=\frac{\mathrm{d} u_{3}}{\mathrm{~d} y}(\xi)$ & -0.1636852 & -0.1433454 & -0.0725202 & -0.00648 \\
$\eta=1 / 4$ & $\mathrm{~d} u_{2}$ \\
$\xi=3 / 4$ & $\frac{\mathrm{d} u_{2}}{\mathrm{~d} y}(\xi)=\frac{\mathrm{d} u_{3}}{\mathrm{~d} y}(\xi)$ & -0.2411004 & 0.1846199 & 0.05934378 & -0.02412919 \\
$\eta=0.49$ & $\frac{\mathrm{d} u_{1}}{\mathrm{~d} y}(\eta)=\frac{\mathrm{d} u_{2}}{\mathrm{~d} y}(\eta)$ & -.002095967 & -0.07054511 & -0.1803686 & -0.2219087 \\
$\xi=0.51$ & $\frac{\mathrm{d} u_{2}}{\mathrm{~d} y}(\xi)=\frac{\mathrm{d} u_{3}}{\mathrm{~d} y}(\xi)$ & -0.02130357 & -0.08492409 & -0.1811111 & -0.1886142 \\
\hline
\end{tabular}

4) For a given transition layer thickness, absolute value of shear stress at each of the lower and upper interfaces increases with increasing $\mathrm{Da}$.

5) The effect of increasing $n$ on the absolute value of shear stress at the interfaces for a given thickness and $D a$ is that at each interface, this absolute value increases with increasing $\mathrm{n}$. This is true for both thin and thick transition layers, and for the range of Da used.

\section{Friction Factor}

A quantity of interest is the negative of the shear stress term at the interface between the channel and the transition layer, namely $c_{f}=-\frac{\mathrm{d} u_{1}}{\mathrm{~d} y}$ at $y=\eta$. This has been analyzed and defined by Nield and Kuznetsov, [1], as a friction factor representing the dimensionless frictional stress in the fluid at the interface. From Equation (62) we can see that

$$
c_{f}=-\frac{\mathrm{d} u_{1}(\eta)}{\mathrm{d} y}=\eta-c_{1} .
$$

Values of $-c_{f}$ for different $D a$, layer thickness and $n=1$ are listed in Table 5 and compared with the values obtained by Nield and Koznetsov, [1], for the same problem. Agreement between the current results and Nield and Koznetsov results, up to the three significant digits they report is clear from Table 5. Values of $-c_{f}$ for different $D a$, layer thickness and $n=2$ are listed in Table 6 .

\section{Mean Velocity across the Layers}

The dimensionless mean velocities across the layers are defined as

$$
\begin{gathered}
\bar{u}_{1}=\int_{0}^{\eta} u_{1} \mathrm{~d} y=\frac{1}{2} c_{1} \eta^{2}+d_{1} \eta-\frac{1}{6} \eta^{3} \\
\bar{u}_{2}=\int_{\eta}^{\xi} u_{2} \mathrm{~d} y=\int_{\eta}^{\xi} c_{2} A_{n}\left(\tau_{n}(y-\eta)\right)+d_{2} B_{n}\left(\tau_{n}(y-\eta)\right) \\
+\frac{\pi}{2 \sqrt{p} \sin (p \pi) M_{2}\left(\tau_{n}\right)^{2}} Z_{n}\left(\tau_{n}(y-\eta)\right) \mathrm{d} y
\end{gathered}
$$


Table 5. Values of $c_{f}$ for different $D a$, layer thickness and $n=1$.

\begin{tabular}{|c|c|c|c|c|c|c|c|}
\hline $\mathrm{Da}$ & & 1 & 0.1 & 0.01 & 0.001 & 0.0001 & 0.00001 \\
\hline $\begin{array}{l}\eta=1 / 3 \\
\xi=2 / 3\end{array}$ & $c_{f}$ & $\begin{array}{c}-0.1538176 \\
-0.153^{\star}\end{array}$ & -0.07977407 & $\begin{array}{c}0.04941739 \\
0.049^{\star}\end{array}$ & 0.1152168 & -0.1436413 & -0.1562024 \\
\hline $\begin{array}{l}\eta=1 / 4 \\
\xi=3 / 4\end{array}$ & $c_{f}$ & -0.2365115 & -0.1575664 & -0.01321413 & 0.06447045 & 0.0981652 & 0.1129008 \\
\hline $\begin{array}{l}\eta=0.49 \\
\xi=0.51\end{array}$ & $c_{f}$ & $\begin{array}{c}0.0022864 \\
0.002^{\star}\end{array}$ & 0.0715832 & $\begin{array}{c}0.1820249 \\
0.182^{\star}\end{array}$ & 0.2237071 & 0.2361822 & 0.2409651 \\
\hline
\end{tabular}

*Nield and koznetsov results.

Table 6. Values of $c_{f}$ for different $D a$, layer thickness and $n=2$.

\begin{tabular}{cccccccc}
\hline & & 1 & 0.1 & 0.01 & 0.001 & 0.0001 & 0.00001 \\
\hline $\begin{array}{l}r=1 / 3 \\
\xi=2 / 3\end{array}$ & $c_{f}$ & -0.156954 & -0.0979064 & 0.0180856 & 0.0860125 & 0.122236 & 0.142053 \\
$\eta=1 / 4$ & & & & & & & \\
$\xi=3 / 4$ & $c_{f}$ & -0.241100 & -0.184620 & -0.0593438 & 0.0241292 & 0.0696405 & 0.0944781 \\
$\eta=0.49$ & & & & & & & \\
$\xi=0.51$ & $c_{f}$ & 0.0020959 & 0.0705451 & 0.180369 & 0.221909 & 0.234224 & 0.239089 \\
\hline
\end{tabular}

By letting $t=\tau_{n}(y-\eta)$ in (7.61) we get

$$
\begin{gathered}
\bar{u}_{2}=\frac{c_{2}}{\tau_{n}} \int_{0}^{\tau_{n}(y-\eta)} A_{n}(t) \mathrm{d} t+\frac{d_{2}}{\tau_{n}} \int_{0}^{\tau_{n}(y-\eta)} B_{n}(t) \mathrm{d} t \\
+\frac{\pi}{2 \sqrt{p} \sin (p \pi) M_{2}\left(\tau_{n}\right)^{3}} \int_{0}^{\tau_{n}(y-\eta)} Z_{n}(t) \mathrm{d} t \\
\bar{u}_{3}=\int_{\xi}^{1} u_{3} \mathrm{~d} y=\int_{\xi}^{1}\left[c_{3} \exp \left(\lambda_{3} y\right)+d_{3} \exp \left(-\lambda_{3} y\right)+\frac{1}{M_{2} \lambda_{3}^{2}}\right] \mathrm{d} y \\
=\frac{1}{\lambda_{3}}\left[c_{3}\left\{\exp \left(\lambda_{3}\right)-\exp \left(\lambda_{3} \xi\right)\right\}-d_{3}\left\{\exp \left(-\lambda_{3}\right)-\exp \left(-\lambda_{3} \xi\right)\right\}+\frac{1-\xi}{M_{2} \lambda_{3}}\right]
\end{gathered}
$$

The dimensionless mean velocity across the configuration (that is, across the three layers) is defined as

$$
\bar{u}=\bar{u}_{1}+\bar{u}_{2}+\bar{u}_{3}=\int_{0}^{\eta} u_{1} \mathrm{~d} y+\int_{\eta}^{\xi} u_{2} \mathrm{~d} y+\int_{\xi}^{1} u_{3} \mathrm{~d} y
$$

and represents a measure of the overall volume flux through the channel configuration.

The above expressions are evaluated using Maple and the values are listed in for $n=1$ and $n=2$ in Table 7 and Table 8, respectively, for different transition layer thicknesses and different $D a$. Comparison is made for the case of $n=1$ with the Nield and Koznetsov results [1]. Agreement between the results is evident from Table 7. It is also evident from Table 7 and Table 8 that increasing $\mathrm{n}$ results in a higher mean velocity across the channel for thick layers but the increase is minimal for thin layers, for a given $\mathrm{Da}$. 
Table 7. Mean velocity across the channel for $n=1$.

\begin{tabular}{cccccc}
\hline Da & & $D a=1$ & $D a=0.1$ & $D a=0.01$ & $D a=0.001$ \\
$\eta=1 / 3$ & $\bar{u}$ & $\begin{array}{c}0.079395 \\
0.0794^{*}\end{array}$ & 0.05692682 & $\begin{array}{c}0.02071123 \\
0.0207^{*}\end{array}$ & 0.007936325 \\
$\xi=2 / 3$ & & & & & \\
$\eta=1 / 4$ & $\bar{u}$ & 0.07938781 & 0.05659447 & 0.01881449 & 0.00553387 \\
$\xi=3 / 4$ & & & & & \\
$\eta=0.49$ & $\bar{u}$ & 0.07940428 & 0.05735128 & 0.02355505 & 0.01315206 \\
$\xi=0.51$ & & $0.0794^{*}$ & & $0.0236^{*}$ & \\
\hline
\end{tabular}

Table 8. Mean velocity across the channel for $n=2$.

\begin{tabular}{|c|c|c|c|c|}
\hline & & $D a=1$ & $D a=0.1$ & $D a=0.01$ \\
\hline $\begin{array}{l}\eta=1 / 3 \\
\xi=2 / 3\end{array}$ & $\bar{u}$ & 0.08744141 & 0.06403606 & 0.02670879 \\
\hline $\begin{array}{l}\eta=1 / 4 \\
\xi=3 / 4\end{array}$ & $\bar{u}$ & 0.09902008 & 0.06954515 & 0.02749235 \\
\hline $\begin{aligned} \eta & =0.49 \\
\xi & =0.51\end{aligned}$ & $\bar{u}$ & 0.07945951 & 0.05759342 & 0.0238459 \\
\hline
\end{tabular}

For a given transition layer thickness and a given value of $n$, the total volume flux decreases with decreasing $\mathrm{Da}$. This is due to flow retardation for smaller permeability.

It should be noted that since the velocity computations for thick layers are accurate for $D a$ as low as 0.001 , so are the computations of the mean velocity.

\section{Velocity Profiles}

Velocity profiles across the three-layered channel have been obtained for the various flow parameters and are graphed in Figures 3-9.

Figure 3 illustrates the velocity profile for a thick transition layer, $\eta=\frac{1}{3}, \xi=\frac{2}{3}$, when $D a=1$ and $n=1$. This velocity profile is parabolic and is close to what one might expect in the case of Poiseuille flow and the solution of the Navier-Stokes equations due to the high value of permeability. This parabolic shape is lost when $D a$ is reduced, as shown in Figure 4, as the flow in upper part of the channel moves slower than in the middle part, which is in turn slower than the Navier-Stokes flow in the lower part of the configuration.

For the case of $n=1$, and thick transition layer, Figure 5 illustrates the effect of changing $\mathrm{Da}$ on the velocity profile. The case of thin layer is shown in Figure 6. Both figures demonstrate a velocity profile distortion with decreasing $\mathrm{Da}$ as the permeability becomes lower in the upper region, resulting in slower flow. The same is illustrated in Figure 7, Figure 8 for the case of $n=2$.

The effect of varying $n$ is illustrated in Figure 9, which demonstrates the relative increase of velocity in the transition layer with increasing $n$. This may be 


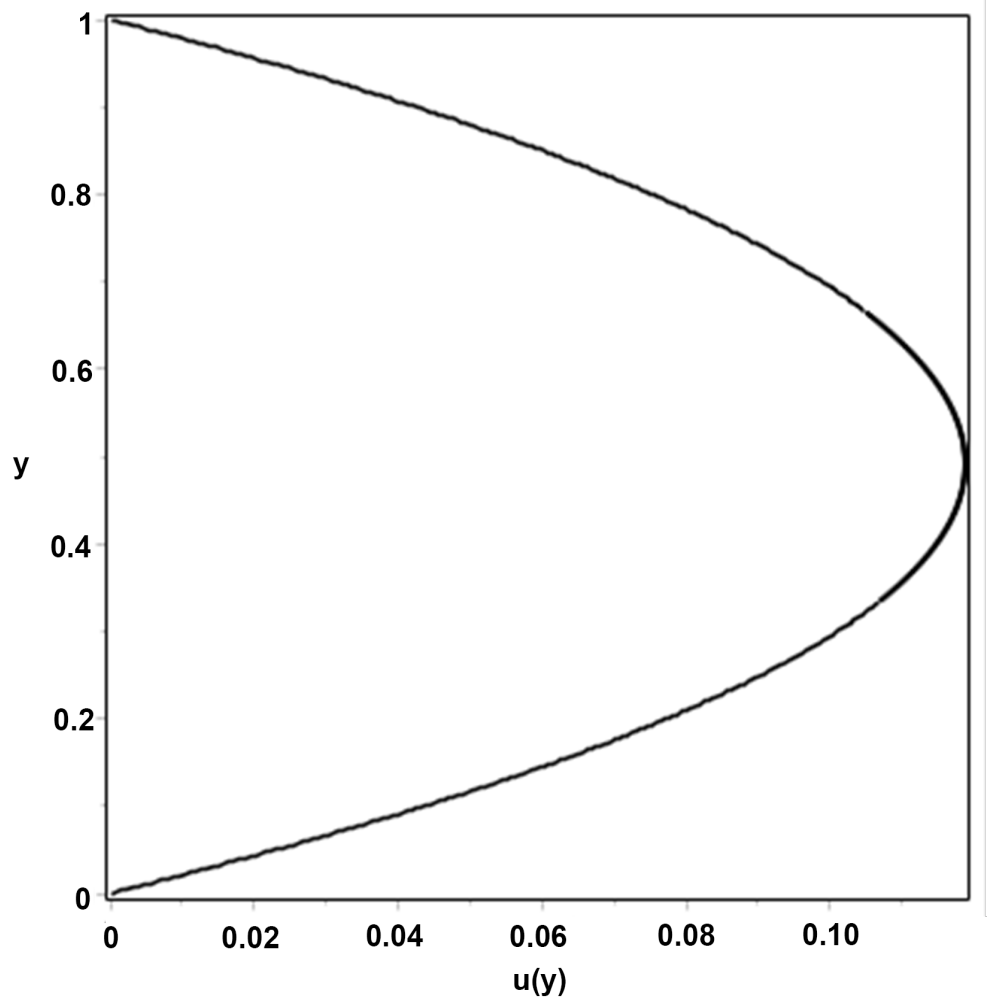

Figure 3. Velocity Profile $\mathrm{u}(\mathrm{y})$, for $D a=1, \eta=\frac{1}{3}, \xi=\frac{2}{3}$, and $n=1$.

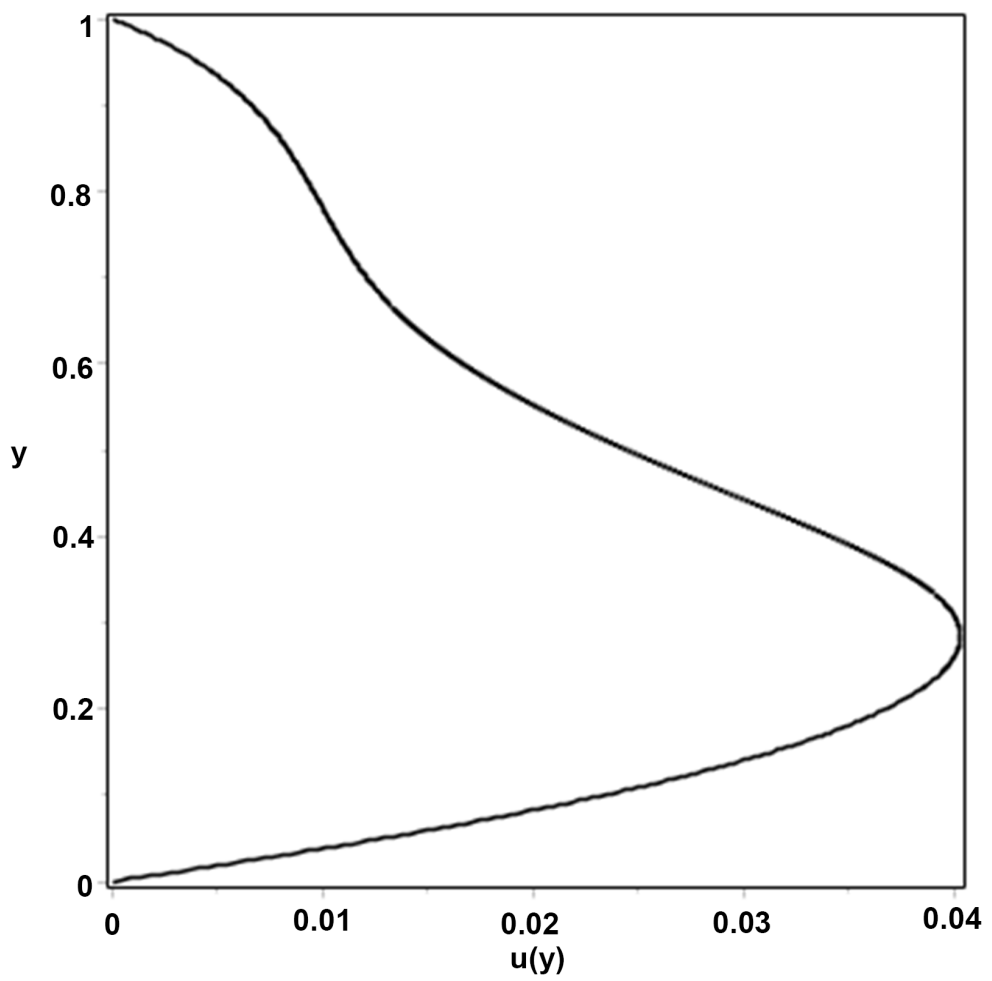

Figure 4. Velocity Profile $\mathrm{u}(\mathrm{y})$, for $D a=0.01, \eta=\frac{1}{3}, \xi=\frac{2}{3}$, and $n=1$. 


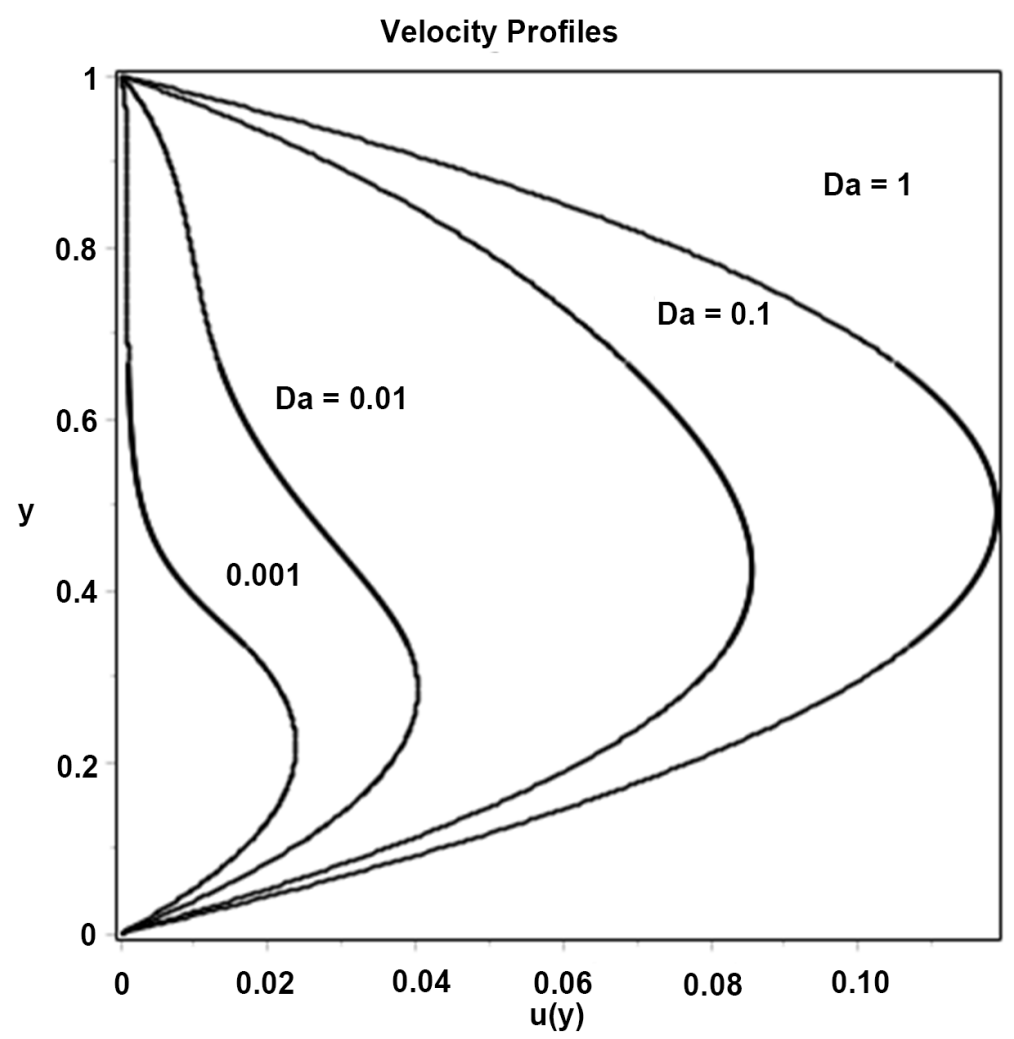

Figure 5. Velocity profiles in the three layers for $n=1, \eta=\frac{1}{3}, \xi=\frac{2}{3}$ and different Da.

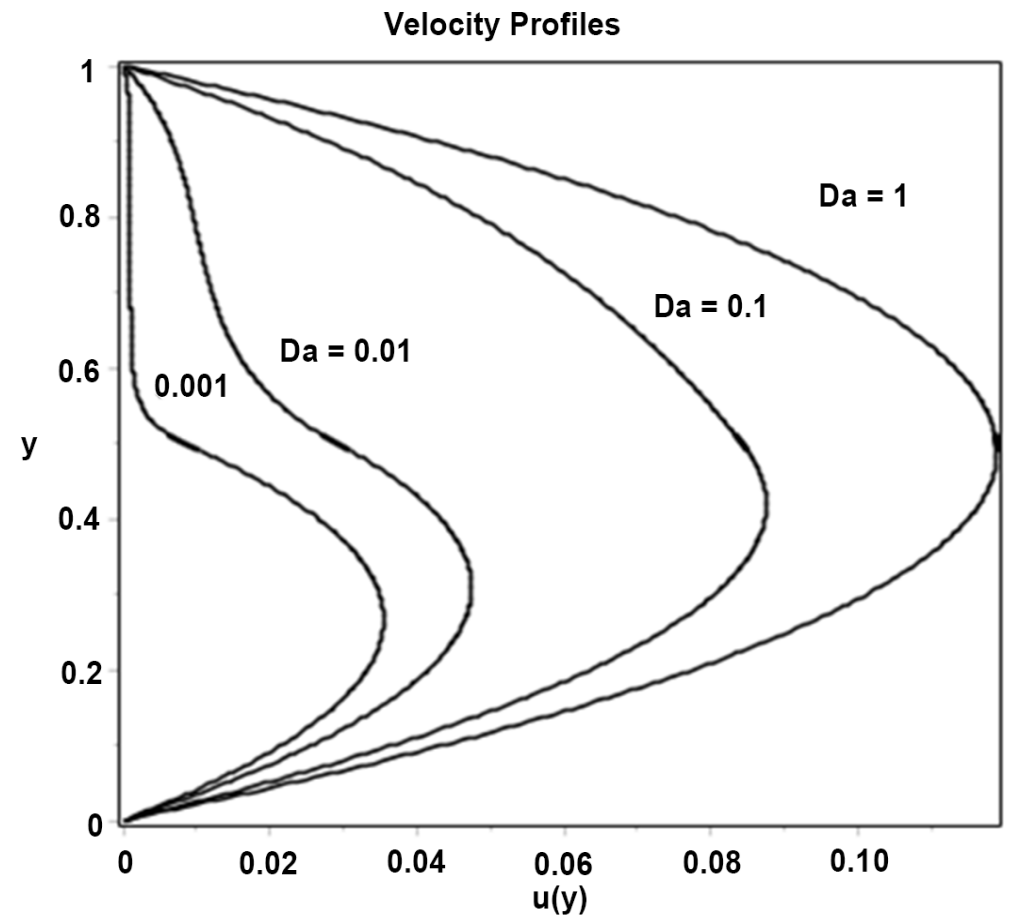

Figure 6. Velocity profiles in the three layers for $n=1, \eta=0.49, \xi=0.51$ and different values of Darcy number. 


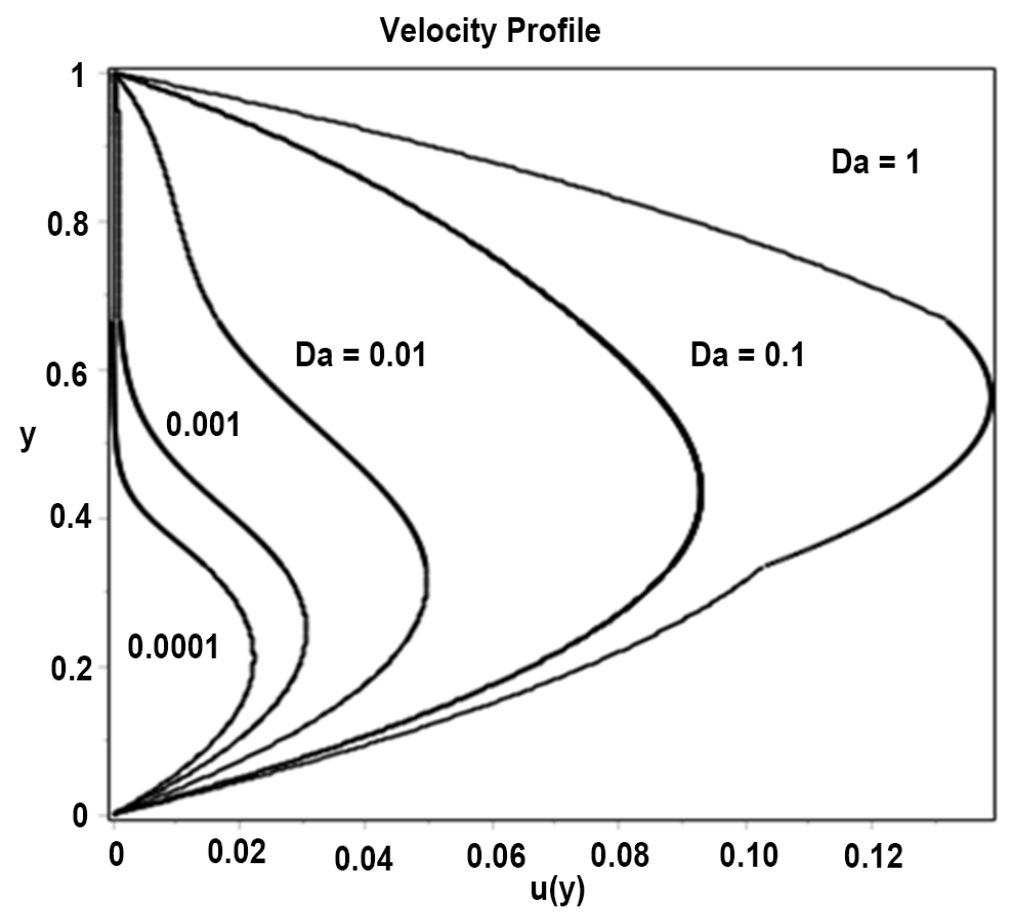

Figure 7. Velocity profiles in the three layers for $n=2, \eta=\frac{1}{3}, \xi=\frac{2}{3}$ and different $D a$.

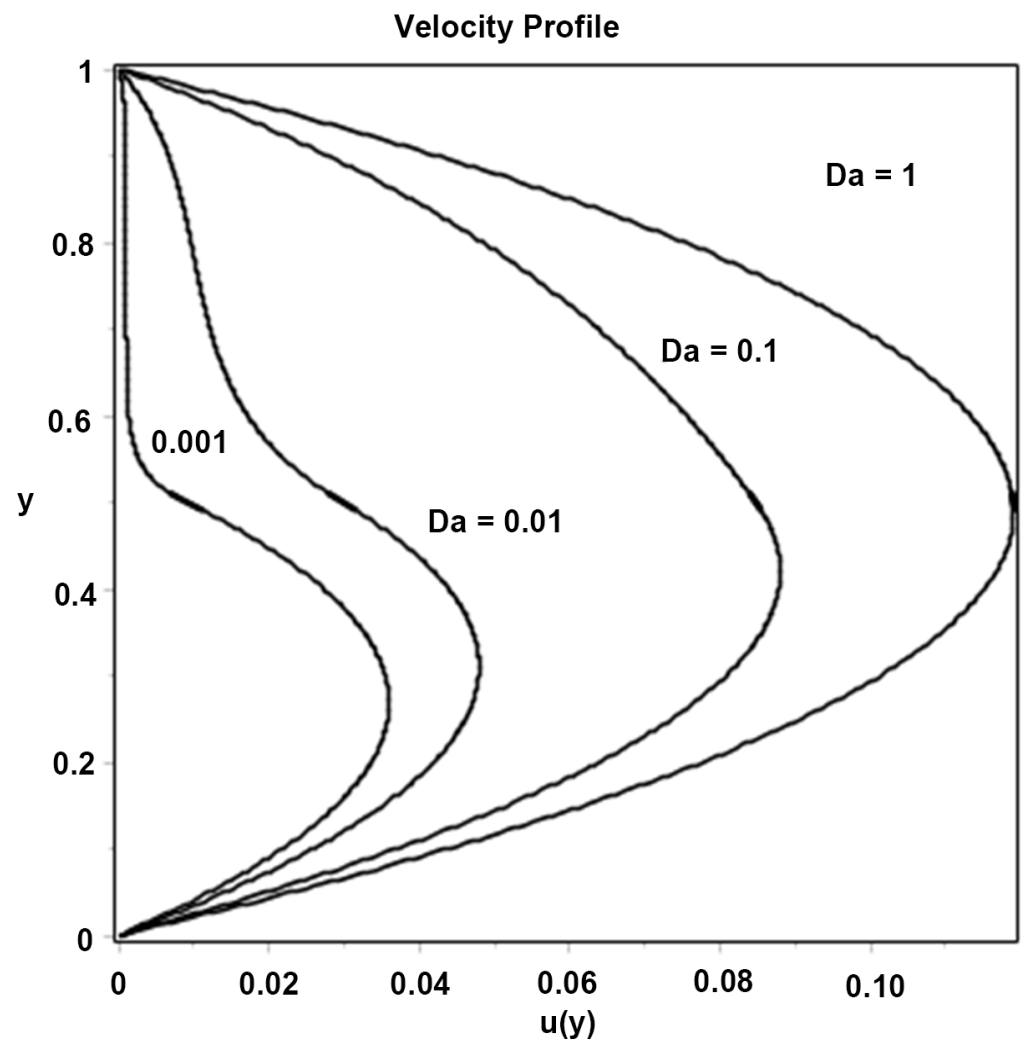

Figure 8. Velocity profiles in the three layers for $n=2, \quad \eta=0.49, \xi=0.51$ and different $D a$. 


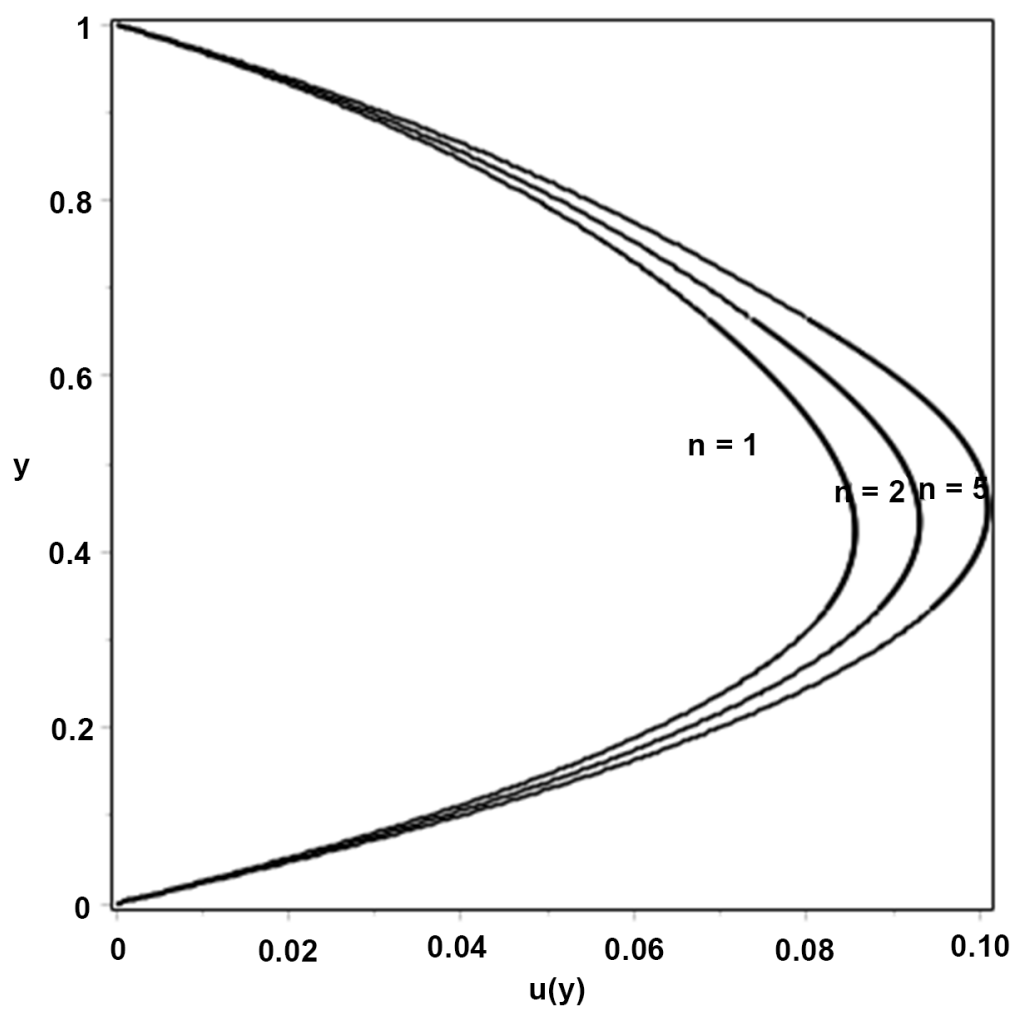

Figure 9. Velocity profiles in the layers for $D a=0.1, \eta=\frac{1}{3}, \xi=\frac{2}{3}$ and different $n$.

attributed to higher momentum transfer from the adjacent layers that tend to increase the flow velocity there.

\section{Conclusion}

In this work, we considered the problem of flow through a porous medium over a free-space channel in the presence of a transition layer. This problem was treated by Nield and Kuznetsov [1] to illustrate the characteristics of the flow when the transition layer is a Brinkman layer of variable permeability described as the reciprocal of a linear polynomial. In this work, we considered the permeability to vary as the reciprocal of an $n$th degree polynomial and to solve the resulting generalized Airy's differential equation. For the case of $n=1$, our results agree with those obtained by Nield and Kuznetsov [1].

\section{References}

[1] Nield, D.A. and Kuznetsov, A.V. (2009) The Effect of a Transition Layer between a Fluid and a Porous Medium: Shear Flow in a Channel. Transport in Porous Media, 78, 477-487. https://doi.org/10.1007/s11242-009-9342-0

[2] Nield, D.A. (1983) The Boundary Correction for the Rayleigh-Darcy Problem: Limitations of the Brinkman Equation. Journal of Fluid Mechanics, 128, 37-46. https://doi.org/10.1017/S0022112083000361

[3] Goyeau, B., Lhuillier, D. and Velarde, M.G. (2003) Momentum Transport at a 
Fluid-Porous Interface. International Journal of Heat and Mass Transfer, 46, 40714081. https://doi.org/10.1016/S0017-9310(03)00241-2

[4] Goharzadeh, A., Khalili, A. and Jørgensen, B.B. (2005) Transition Layer Thickness at a Fluid-Porous Interface. Physics of Fluids, 17, 057102.

https://doi.org/10.1063/1.1894796

[5] Parvazinia, M., Nassehi, V., Wakeman, R.J. and Ghoreishy, M.H.R. (2006) Finite Element Modelling of Flow through a Porous Medium between Two Parallel Plates Using the Brinkman Equation. Transport in Porous Media, 63, 71-90. https://doi.org/10.1007/s11242-005-2721-2

[6] Chandesris, M. and Jamet, D. (2007) Boundary Conditions at a Fluid-Porous Interface: An a priori Estimation of the Stress Jump Boundary Conditions. International Journal of Heat and Mass Transfer, 50, 3422-3436. https://doi.org/10.1016/j.ijheatmasstransfer.2007.01.053

[7] Hill, A.A. and Straughan, B. (2008) Poiseuille Flow in a Fluid Overlying a Porous Medium. Journal of Fluid Mechanics, 603, 137-149. https://doi.org/10.1017/S0022112008000852

[8] Duman, T. and Shavit, U. (2010) A Solution of the Laminar Flow for a Gradual Transition between Porous and Fluid Domains. Water Resources Research, 46, 09517. https://doi.org/10.1029/2009WR008393

[9] Tao, J., Yao, J. and Huang, Z. (2013) Analysis of the Laminar Flow in a Transition Layer with Variable Permeability between a Free-Fluid and a Porous Medium. Acta Mechanica, 224, 1943-1955. https://doi.org/10.1007/s00707-013-0852-Z

[10] Hamdan, M.H. and Kamel, M.T. (2011) On the Ni(x) Integral Function and Its Application to the Airy's Non-Homogeneous Equation. Applied Mathematics and Computation, 217, 7349-7360. https://doi.org/10.1016/j.amc.2011.02.025

[11] Nield, D.A. and Bejan, A. (2006) Convection in Porous Media. 3rd Edition, Springer.

[12] Nield, D.A. (2009) The Beavers Joseph Condition and Related Matters: A Historical and Critical Note. Transport in Porous Media, 78, 537-540. https://doi.org/10.1007/s11242-009-9344-y

[13] Swanson, C.A. and Headley, V.B. (1967) An Extension of Airy's Equation. SIAM Journal on Applied Mathematics, 15, 1400-1412. https://doi.org/10.1137/0115123 